\title{
Soft fiber-reinforced pneumatic actuator design and fabrication: Towards robust, soft robotic systems *
}

\author{
Jan Fras $^{1}$ and Kaspar Althoefer ${ }^{1}$ \\ Centre for Advanced Robotics @ Queen Mary (ARQ), Faculty of Science and \\ Engineering, Queen Mary University of London \\ j.fras@qmul.ac.uk
}

\begin{abstract}
Soft robotics is a rapidly evolving, young research area. So far there are no well-established design standards nor fabrication procedures for soft robots. A number of research groups are working on soft robotics solutions independently and we can observe a range of designs realized in different ways. These soft robots are based on various actuation principles, are driven with various actuation media, and offer various actuation properties. Still, most of them require lots of manual effort and high manual fabrication skills from the person manufacturing these kinds of robots. A significant share of the proposed designs suffers from some imperfections that could be improved by simple design changes. In this work, we propose a number of design and fabrication rules for improving the performance and fabrication complexity of soft fiber-reinforced pneumatic actuators. The proposed design approach focuses on a circular geometry for the pressure chambers and applying a dense, fiber-based reinforcement. Such an approach allows for a more linear actuator response and reduced wear of the actuators, when compared to previous approaches. The proposed manufacturing procedure introduces the application of the reinforcement before the fabrication of the actuator body, significantly reducing the required fabrication effort and providing more consistent and more reliable results.
\end{abstract}

Keywords: Soft robotics, soft pneumatic actuation, soft actuation, soft robotics, soft robotics manufacturing, compliant robotics.

\section{Introduction}

Soft robotics is a young and very promising research area. Soft robots are compliant, they can passively adapt to the environment and safely interact with fragile objects. In many cases, they also consist of fewer parts than traditional,

\footnotetext{
* This work was supported in part by the EPSRC in the framework of the NCNR (National Centre for Nuclear Robotics) project (EP/R02572X/1) and the Innovate UK funded and q-bot led project WormBot.
} 
rigid equivalents and provide very good power-to-weight and force-to-weight ratio [1]. They also promise an opportunity to simplify the control strateges when compared to traditional robots [2]. For those reasons they are considered to be suitable for many applications such as medical applications (minimal invasive surgery [3], laparoscopy [4], rehabilitation [5]) a human-robot cooperation [6, 7], grasping $[8]$ or prosthetics $[9,10]$. There are plenty of actuators that offer various actuation principles and motion types. They are fabricated using various materials and techniques. The design space of soft actuators is very broad and thus still not fully explored. Despite such a big interest in soft robotics, many pneumatic system designs are suboptimal and fabricated in an inefficient way.

In fact, design and manufacturing are big issues in soft robotics [11]. Due to the short history, there are no well-defined standards for soft robot designs yet. There are various designs of soft actuators and various manufacturing procedures [11]. Most techniques require a lot of manual operations and high manual skills from the person manufacturing such robots. The most commonly used technique is molding and usually consists of many molding steps to manufacture the device. In case any reinforcement is required, it is usually achieved requiring additional manual manufacturing steps.

Researchers explore additive techniques extensively as they would to minimize required labor and improve repeatability, reliability, and prototyping speed $[12,13]$. However, those techniques are still very limited regarding the material selection (in particular when different materials within the process are required) and struggle with the fabrication of airtight internal voids.

In this paper, we propose some general rules to be considered when designing new soft-robotics systems in order to make them more reliable, their actuation response more linear and fabrication more convenient. For the purpose of this paper, we will focus only on soft fiber-reinforced actuators driven with positive pneumatic pressure.

The paper is organized as follows: In section section 2 we discuss the general design rules that apply to fiber-based reinforcement section 2.1 and the actuator geometry section 2.2 . In section section 3 we discuss the manufacturing of the proposed actuators with the focus on the reinforcement and the actuator body. In section section 4 we conclude the paper.

\section{The soft actuator design}

There are various actuation types and each of them can be implemented by different actuator designs. The most basic actuation types are linear actuation (expansion, contraction), bending and twisting. The linear actuator design can be considered trivial since any airtight tube made of flexible material can be considered as a linear actuator. Adding a braided sheath converts it to a contracting McKibben pneumatic muscle [14]. Adding a fiber reinforcement perpendicular to its main axis makes it a linearly expanding actuator. Further addition of strain limiting layers or fibers at certain angles can convert it to a bending or twisting actuator. Bending and twisting motion can be also induced by the geometry of 
the actuator such as unsymmetrical actuation chambers or a curved, toroidal shape.

\subsection{Reinforcement}

In the case of positive-pressure-driven soft actuators, their deformation is caused by the internal pressure applied to a pressure chamber. The pressure acting on the internal chamber surfaces induces forces that stretch the device. Since a pressure inside a closed volume in a passive state can be considered to be constant, those forces are distributed equally in the whole actuation chamber volume. For that reason, the actuator tends to deform not only in the desired direction but in all the other directions as well. In many applications, such deformations are very undesired. Thus, pneumatic actuators often contain some reinforcement, some structures that are designed to amplify the desired motion and to limit any other deformation of the device. Those structures can be made of the same material the actuator is made of (e.g., bellows or groves) or different material (such as fibers, fabrics and tendons). In all those cases, the reinforcement limits the deformation in some direction while allowing it in the direction of actuation. In our work, we focus on fiber-based reinforcement which has a number of advantages. First of all, the fibers can have a very small diameter, so that they can be embedded into the device without greatly affecting its dimensions or geometry. They can also be very resilient allowing high pressure to be applied into the device and consequently to exert high external forces. Ideally flexible fibers cannot resist any forces in the radial direction but they do restrict elongation. Thanks to that, they do not affect the overall stiffness of the device, but only the stretch along the direction they are oriented. Fiber-based reinforcement can be described by various features such as the material used for it, its geometry in the chamber cross-section and its density along the primary axis (how close are the fibers each other). The material of the fiber does not affect the mechanics of the actuator that much, since we consider it to be ideally flexible, so let's discuss the other two features.

The reinforcement density The fibers embedded into the silicone constrain the silicone deformation in the direction along the fiber. Since the silicone is flexible and stretchable, this effect is the strongest in the close neighborhood of the fiber and gets lower when moving away from the fiber. Consider a cylindrical actuator reinforced with circles made of a thread as depicted in Figure 1.

Distances between the reinforcement rings grow when the actuator is pressurized. In case the initial reinforcement density is sparse (Figure 1a) it will get even sparser during the actuation allowing the pressure to expand the chamber in between the reinforcement (ballooning). Making the reinforcement denser improves the situation, as smaller gaps between threads restrict the ballooning better. Ballooning is a problem, as this kind of inflation induces a nonlinear behavior in the actuator, leads to more intensive wear of the flexible material in the areas it expands more (balloons) and results in lower pressure resistance of the device. 


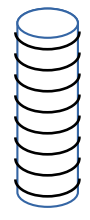

(a)

(a)

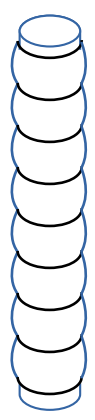

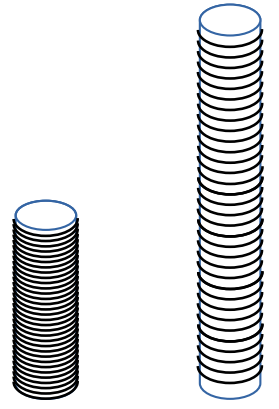

(b)

Fig. 1: Comparison of sparse and dense reinforcement applied to a linear actuator. Sparse reinforced actuator (a) expands between the reinforcing rings, while dense reinforced (b) does not.

Such a principle applies to all the expanding actuators including linear and rotational ones, see Figure 2.
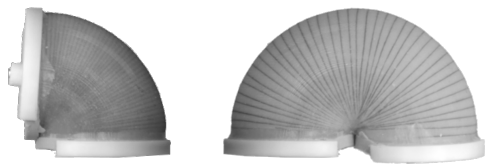

(a)
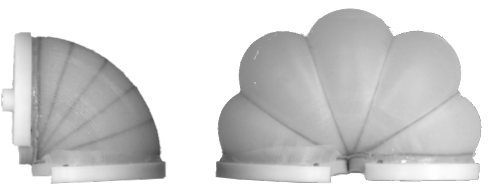

(b)

Fig. 2: Different angular densities of reinforcing fibers. Ballooning visible for the spare reinforcement. (a) dense reinforced actuator in passive state and actuated, (b) sparse reinforced actuator passive and actuated

On the other hand, the ballooning causes the actuation area to grow in parallel with the pressure and consequently to amplify the actuation response of the actuator. One can argue that this way we can eventually get an actuator that can generate higher forces and extend and bend more at the same input pressure. However, ballooning means the actuator expands its radial dimensions during the actuation. Thus, if the higher forces are required, a larger actuator can be used so that the higher force will be exerted while keeping the benefits of the dense reinforcement (linearity, reliability, durability).

An example of how the reinforcement density affects the actuation process is presented in Figure 3. 


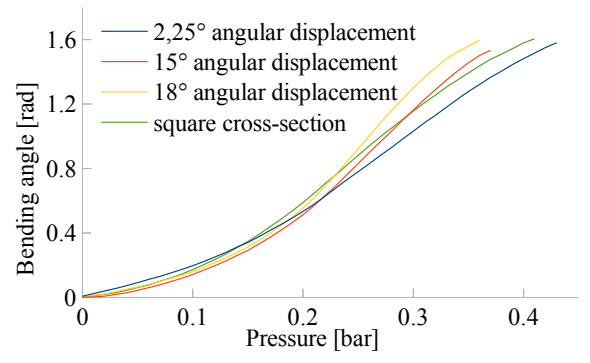

(a)

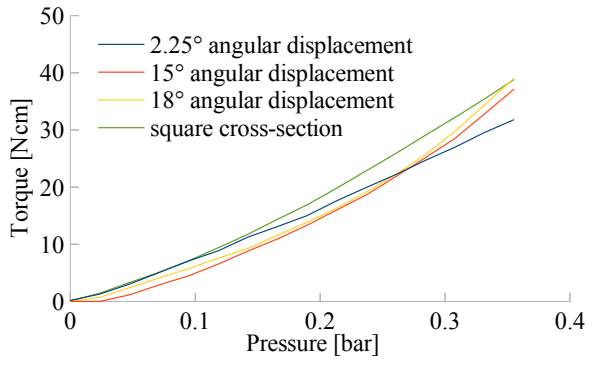

(b)

Fig. 3: The characteristics of a bending actuator for different reinforcement densities. (a) bending angle vs pressure, b) generated torque vs pressure [15].

The reinforcement pitch Most commonly, the reinforcement of fiber-reinforced actuators is made with a single thread. This means that there are no individual rings, but subsequent periods of a helix made with a single thread. Thus the actual overall shape of the reinforcement is helical and the thread is not perpendicular to the actuator axis, but declined by some angle $\alpha$, Figure 4 . It is important to note that value of angle $\alpha$ is not constant and changes while the actuator elongates.
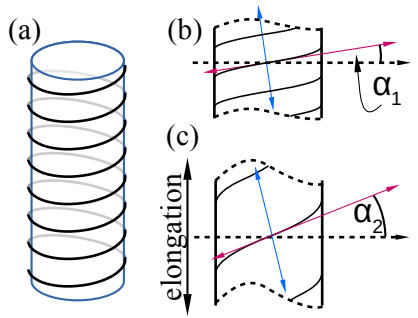

Fig. 4: The reinforcement in most fiber-reinforced actuators has a helical geometry. (a) The more spare the reinforcement is, the bigger value of the pitch angle $(\alpha)$. (b) The pitch angle determines the actuator behaviour. The deformation is constrained only in the direction of the fiber (red axis) and not affected in the perpendicular direction (blue axis). (c) The elongation causes the pitch angle to increase, so the effect increases as well.

As already mentioned, the fibers can constrain only expansion in the direction parallel to them. Since they are angled, the tension they generate is not perpendicular to the actuation axis. In such a case, they not only constrain the radial expansion but also cause the actuator to twist, see Figure 5. Consider an imaginary cut line along the actuator's pressure chamber. If the chamber is cut along this line and resulting membrane is put flat on a surface, it contains a 
set of straight sections of fibers parallel to each other. All of them are angled resulting from the helical shape of the reinforcement. Since only the expansion in the direction parallel to fibers is constrained, if a uniform stretching net force is applied to the membrane it would deform in a way it is no longer rectangular. The deformation direction will be perpendicular to the fibers, depicted as green arrow in Figure 5. If the membrane is rolled again to create the chamber back and stitched along the cutting line, we will notice that the cutting line is no longer straight, and beside the elongation the actuator has twisted a bit.

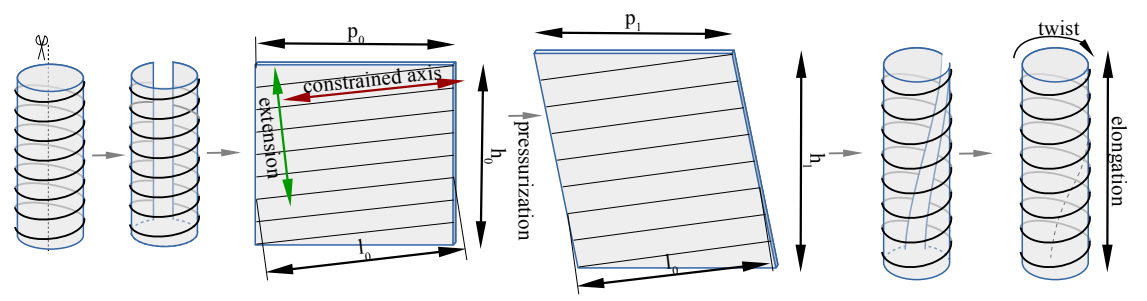

Fig. 5: Explanation for twisting motion resulting from angled fibers.

The relation of the elongation and twist of the device could be described by $\sin (\alpha)$ and $\cos (\alpha)$ functions of $\alpha$ angle. While the elongation of the actuator along its primary actuation axis is proportional to $\cos (\alpha)$ function, its twist is proportional to $\frac{\sin (\alpha)}{r}$, where $r$ is radious of the actuator's chamber. It is important to notice that single helical reinforcement will never provide pure extension since $\alpha$ can not be zero. Some twisting will always appear. However, by increasing the reinforcement density we can reduce $\alpha$ so that $\sin (\alpha)<<\cos (\alpha)$, and that way reduce the twisting motion to an acceptable level. In some cases twist might be a desired behavior [16-18], but often it is not, and require an additional helix of the reinforcement in the opposite direction to compensate $[9$, 19]. For that reason, the dense reinforcement has one more advantage to consider.

\subsection{The actuation chamber cross-section geometry}

The actuation of soft actuators is based on the deformation of its soft body. For that reason, the cross-section shape of the actuator chamber can change during the actuation process. Since we consider fiber-reinforced actuators, the cross-section of the actuation chamber is constrained by fibers. That said, its perimeter length is constant and has the same value for passive and actuated state. Since the pressure inside the actuation chamber acts to increase the internal volume, the cross-section of the chamber will converge towards a shape that offers biggest area under the constant perimeter constrain. Any geometry of the pressure chamber cross-section will converge towards a circular shape when pressurized and the only geometry that will not change its shape is the circular one, see Figure 6. 


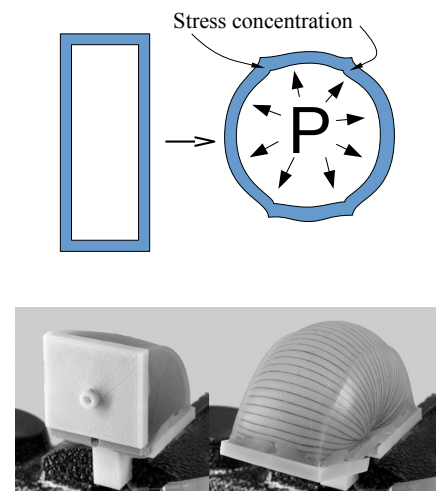

(a)
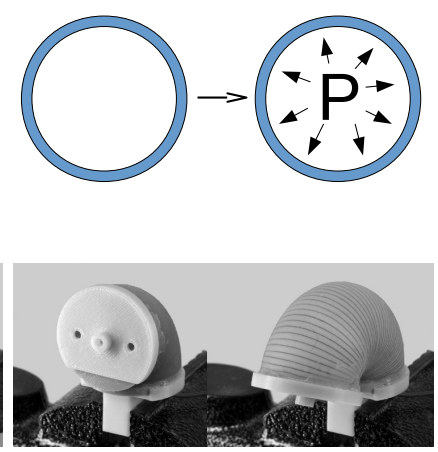

(b)

Fig. 6: Process of pressure chamber deformation during pressurization. (a) rectangular cross-section converges towards circular when pressurized, cross-section area grows, (b) circular cross-section does not change when pressurized, area remains constant.

As a consequence of such deformation, the cross-section area as well as the centroid change, resulting in nonlinearity in the actuation process. Moreover, the device suffers from high strains in corners that in turn may result in fast wear of the material in those areas.

Even if the material wear is not a big issue, it is good to take it into account when designing a soft device. In some applications, the cross-section deformation might cause serious issues with regards to actuation and also sensing [20].

\section{Fabrication}

Most of the fabrication techniques for soft actuators reinforced with fibers require many manual operations. Manual operations are not desired because they are often a source of inconsistency and errors, as well as, labor is a very expensive factor in production. For that reason, we propose some improvements to reduce the manual work required to manufacture the device and to increase the process reliability.

\subsection{The reinforcement}

Conceptually the simplest and the most popular way to reinforce a soft actuator with fibers is to create its body, and put the reinforcement on it afterward. This is, however, the most time and manual-work consuming approach. Moreover, applying the non-stretchable fiber on the soft body of the actuator causes internal tensions in the soft material and thus requires a lot of attention during the 
manufacturing process. The internal tensions are not a big issue for sparse reinforcements, but they became a real problem if the reinforcement has to be dense, Figure 7 . The tension required to wind the actuator with the fiber squeezes the soft body of the actuator and pushes the squeezed material to the sides. When the reinforcement is sparse this might not be considered as a significant problem as the additional material has a lot of space between the fibers to dissipate, Figure $7 \mathrm{a}$. When the reinforcement is dense, however, and the fibers are close to each other the material is pushed forward ((1) in Figure $7 \mathrm{~b})$ to a point where the internal stress moves the fibers apart and pushes soft material out between them ((2) in Figure 7b). This often results in inconsistency of the reinforcement and might likely be a reason for later failure.

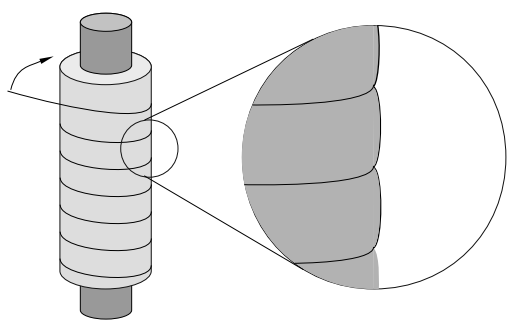

(a)

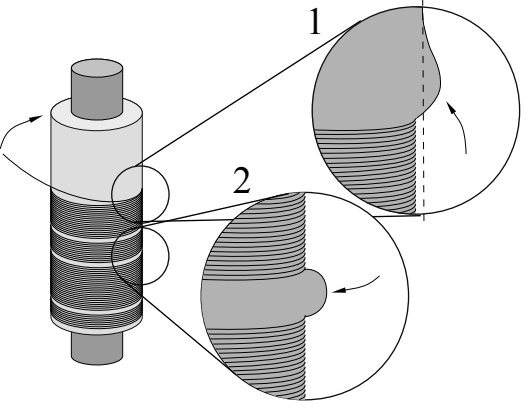

(b)

Fig. 7: Most commonly used technique: manual thread winding on the surface of soft actuator body. (fig. 7a) spare reinforcement, pressed material dissipates between fibers, (fig. 7b) dense reinforcement, pressed material is pushed in the direction of winding (1) which leads to potential inconsistencies in the reinforcement (2).

For that purpose, we propose to reverse this order and create the reinforcement first, before the soft acutor's body is made. For that purpose, a rigid moulding core can be used. Thanks to that there is no deformation induced by the fiber's tension. Using a rigid rod for that purpose allows also to automatize the process easily Figure 8.

Such an approach is not limited to cylindrical actuation chambers. In $[21,10]$ we proposed a double-chamber conical actuator capable of varying the bending direction and in [15] we proposed a rotary actuation with toroidal actuation chamber. However, in case the actuator has axial symmetry, the reinforcement can be easily deployed by rotating the rod (Figures $8 \mathrm{a}$ and $8 \mathrm{~b}$ ). In such a case, a screw gun or a dedicated winding machine can be used. Toroidal reinforcement (Figure 8c), however, still needs to be made by hand. 


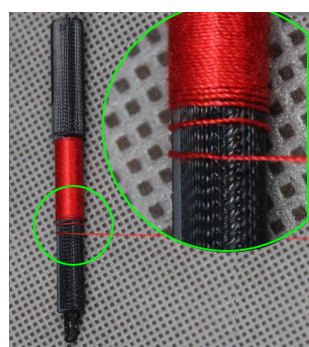

(a)

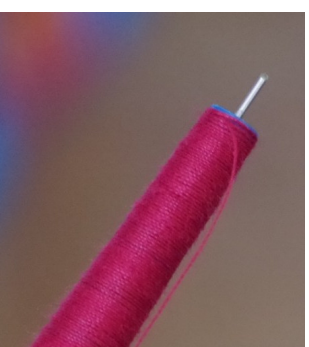

(b)

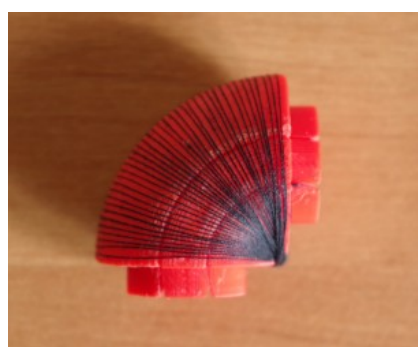

(c)

Fig. 8: The reinforcement created dedicated moulding cores. (a) three-part cylindrical rod, (b) conical core (single-part), (c) three-part toroidal core.

\subsection{The soft body of the actuator}
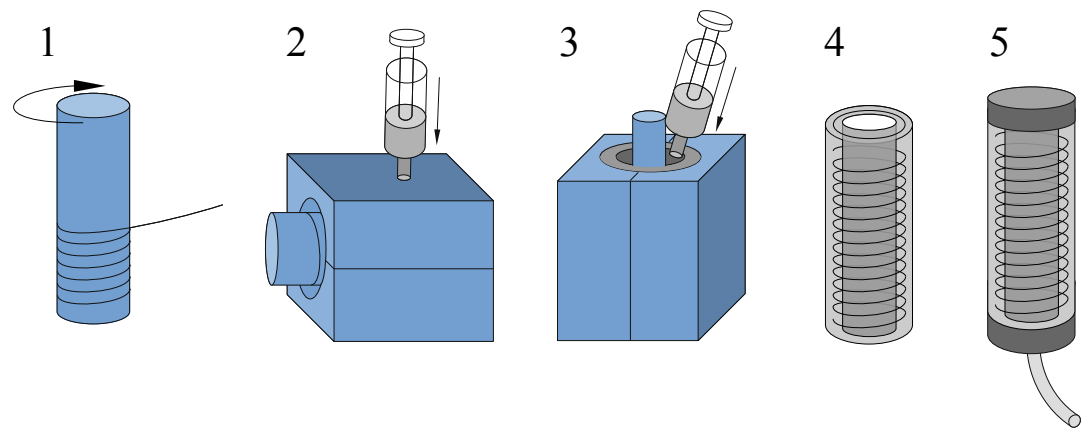

Fig. 9: The actuator fabrication stages. 1) reinforcement winding using a rigid rotating core, 2) external silicone layer casting, 3) internal silicone layer casting (preventing the reinforcement from being detached from the outer layer), 4) the actuation chamber ready, 5) chamber enclosure and pressure pipe attachment.

After the fibers are wound onto the rods, they are covered with silicone material and then removed. To protect the reinforcement structure and to make the removal easier, the employed cylindrical rods are split into three parts. The internal parts are being removed first (while the external parts are still attached to the reinforcement). After the first part is removed, the other two are getting lose and can be removed without any problem. The same procedure can be applied to a bending actuator. Since the adhesion forces in case of silicone are low, and friction between fibers and the internal rod high, pulling it out at once could damage the reinforcement by dragging the threads out of the pressure chamber. This issue can be also solved by using some smooth rods (e.g, polished 
metallic rods) or conical rods (they can be easily removed by pulling out the wider end). Metallic rods are a good solution for cylindrical actuators having some standard dimensions. Other techniques, 3D printing in particular, allow for much more flexible designs like curved shapes, variable diameters etc. In the case of 3D printed parts splitting the core makes the fabrication process easier. After removing the internal molding cores used to shape the reinforcement, smaller core is inserted into the actuator and the volume between the fibers and the core is filled with new silicone material. The new silicone material creates the actual pressure chamber wall. After the material has cured, all the molded parts are removed and the actuator is closed on both ends by dip molding or by gluing on caps made form stiffer silicone. The manufacturing procedure is presented in Figure 9 and photos showing subsequent stages of the actuator being produced in Figure 10

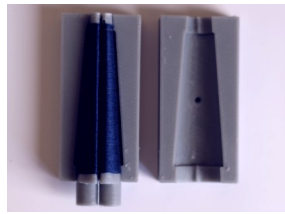

(a)

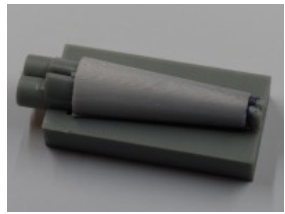

(b)

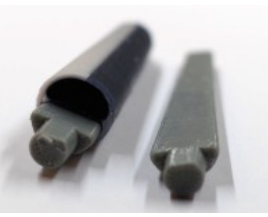

(c)

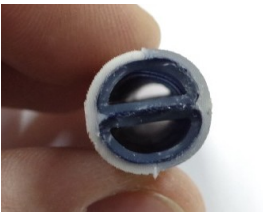

(d)

Fig. 10: Fabrication steps: (a) - reinforcement, (b) - external layer, (c) - reinforcement attached to external layer exposed, (d) - internal chamber layers [22].

\section{Conclusions}

In this paper, we proposed some new rules for soft actuator design and manufacturing. The design rules are built assuming the more linear the actuation the better, but they also help to improve the life span of the actuators by reducing wear. The proposed fabrication guidelines are complementary to the design and help to increase the reliability and repeatability of the actuators, but also reduce the required manual work substantially. The points that need to be considered according to our experience and study are as follows:

1. Always using a circular cross-section will lead to
(a) a more linear response

(b) reduced wear and tear

2. Ensuring a dense fiber reinforcement will lead to

(a) a more linear motion behaviour

(b) reduced wear and tear

(c) reduced twist while keeping simple design (single reinforcing helix)

3. Using rigid shafts for the fiber reinforcement manufacturing will achieve

(a) a more reliable and predictable fabrication process 
(b) less manual effort

The described solutions address the issues we encountered in our research, but those issues are also widely noticeable in the majority of similar designs described in the literature.

\section{References}

1. Yoel Shapiro, Alon Wolf, and Kosa Gabor. Bi-bellows: Pneumatic bending actuator. Sensors and Actuators A: Physical, 167(2):484-494, 2011.

2. K Althoefer. Neuro-fuzzy path planning for robotic manipulators, 1996.

3. Jelizaveta Konstantinova, Ali Shafti, and Kaspar Althoefer. Soft and Stiffnesscontrollable Robotics Solutions for Minimally Invasive Surgery: The STIFF-FLOP Approach. River Publishers, 2018.

4. A. Arezzo, Y. Mintz, M. E. Allaix, S. Arolfo, M. Bonino, G. Gerboni, M. Brancadoro, et al. Total mesorectal excision using a soft and flexible robotic arm: a feasibility study in cadaver models. Surgical Endoscopy, pages 1-10, 2016.

5. Agostino Stilli, Arianna Cremoni, Matteo Bianchi, Alessandro Ridolfi, Filippo Gerii, Federica Vannetti, Helge A Wurdemann, Benedetto Allotta, and Kaspar Althoefer. Airexglovea novel pneumatic exoskeleton glove for adaptive hand rehabilitation in post-stroke patients. In 2018 IEEE International Conference on Soft Robotics (RoboSoft), pages 579-584. IEEE, 2018.

6. Kaspar Althoefer. Antagonistic actuation and stiffness control in soft inflatable robots. Nature Reviews Materials, 3(6):76, 2018.

7. A. Stilli, H. A Wurdemann, and K. Althoefer. A novel concept for safe, stiffnesscontrollable robot links. Soft Robotics.

8. Filip Ilievski, Aaron D Mazzeo, Robert F Shepherd, Xin Chen, and George M Whitesides. Soft robotics for chemists. Angewandte Chemie, 123(8):1930-1935, 2011.

9. Raphael Deimel and Oliver Brock. A novel type of compliant and underactuated robotic hand for dexterous grasping. The International Journal of Robotics Research, 35(1-3):161-185, 2016.

10. J. Fras and K. Althoefer. Soft biomimetic prosthetic hand: Design, manufacturing and preliminary examination. In 2018 IEEE/RSJ International Conference on Intelligent Robots and Systems (IROS), pages 1-6. IEEE, 2018.

11. François Schmitt, Olivier Piccin, Laurent Barbé, and Bernard Bayle. Soft robots manufacturing: a review. Frontiers in Robotics and AI, 5:84, 2018.

12. S. Walker, O.D. Yirmibeolu, U. Daalkhaijav, and Y. Meng. 14 - additive manufacturing of soft robots. In Robotic Systems and Autonomous Platforms, pages 335 359. Woodhead Publishing, 2019.

13. TJ Wallin, J Pikul, and RF Shepherd. 3d printing of soft robotic systems. Nature Reviews Materials, page 1, 2018.

14. Glenn K. Klute and Blake Hannaford. Fatigue characteristics of mckibben artificial muscle actuators. In IROS, 1998.

15. J. Fras, Y. Noh, H Wurdemann, and K. Althoefer. Soft fluidic rotary actuator with improved actuation properties. In International Conference on Intelligent Robots and Systems. IEEE, 2017.

16. Kevin C Galloway, Panagiotis Polygerinos, Conor J Walsh, and Robert J Wood. Mechanically programmable bend radius for fiber-reinforced soft actuators. In Advanced Robotics (ICAR), 2013 16th International Conference on, pages 1-6. IEEE, 2013. 
17. Fionnuala Connolly, Panagiotis Polygerinos, Conor J. Walsh, and Katia Bertoldi. Mechanical programming of soft actuators by varying fiber angle. Soft Robotics, 2(1):26-32, March 20152015.

18. Joshua Bishop-Moser, Girish Krishnan, Charles Kim, and Sridhar Kota. Design of soft robotic actuators using fluid-filled fiber-reinforced elastomeric enclosures in parallel combinations. In Intelligent Robots and Systems (IROS), 2012 IEEE/RSJ International Conference on, pages 4264-4269. IEEE, 2012.

19. Panagiotis Polygerinos, Zheng Wang, Johannes TB Overvelde, Kevin C Galloway, Robert J Wood, Katia Bertoldi, and Conor J Walsh. Modeling of soft fiberreinforced bending actuators. IEEE Transactions on Robotics, 31(3):778-789, 2015.

20. J. Fras, J. Czarnowski, M. Macias, J. Glowka, M. Cianchetti, and A. Menciassi. New stiff-flop module construction idea for improved actuation and sensing. In International Conference on Robotics and Automation, pages 2901-2906. IEEE, 2015.

21. J. Fras, Y. Noh, Macias M., H Wurdemann, and K. Althoefer. Bio-inspired octopus robot based on novel soft fluidic actuator. In submitted to International Conference on Robotics and Automation. IEEE, 2018.

22. J. Fras, M. Macias, Y. Noh, and K. Althoefer. Fluidical bending actuator designed for soft octopus robot tentacle. In 2018 IEEE International Conference on Soft Robotics (RoboSoft), pages 253-257. IEEE, 2018. 